\title{
GPU accelerated circuit analysis using machine learning-based parallel computing model
}

\author{
Shital V. Jagtap ${ }^{1}$ Y. S. Rao ${ }^{2}$
}

Received: 1 September 2019 / Accepted: 1 April 2020 / Published online: 11 April 2020

(c) Springer Nature Switzerland AG 2020

\begin{abstract}
Circuit simulators have the capability to create virtual environment to test circuit design. Simulators save time and hardware cost. However, when components in circuit design increase, most simulators take longer time to test large circuit design, in many cases days or even weeks. Therefore, to handle large dataset and accurate performance, simulators need to be improved. In this paper, we propose machine learning-based parallel implementations of circuit analyser on graphics card with Compute Unified Device Architecture (CUDA). After parsing netlist file, the first approach is to analyse compute intensive mathematical functions and then convert it into parallel executable version. Further, we propose a Design-Level Parallelism with hybrid parallel implementation of components and processing methods. Dynamic decision-making is required to select functions and parameters to map on Graphics Processing Unit (GPU). To reduce load overhead, machine learning clustering approach has been adopted. Combination of procedure clustering and mapping takes few cycles but overall performance enhances efficiency as compared to serial processing.
\end{abstract}

Keywords GPU · Transient analysis · Clustering · LU decomposition · Parallel computation

\section{Introduction}

Circuit design, analysis, and validation play inseparable roles in electronic industries, academics, and in research. Several software simulators are available using which researchers can test circuit possibilities to save time and hardware costs. Added to this is the Internet of Things (loT) that empowers analytical and testing process for the circuit design.

Typically, a good number of simulation software tools are available for circuit design, but for simulation of large circuit, there is still a dearth of good tools [1]. Several hundreds of components are required for simulating large circuit for testing over a long period of time, running for days or a week together. Needless to underline that with the increasing number of components in large circuit, testing time for simulation is bound to increase proportionally. Although there are especially distributed systems or supercomputers to expedite simulation time, they are costly making it unaffordable for general purpose and common users.

To resolve this issue, this research proposes a clusterdriven Graphics Processing Unit (GPU)-based parallel computing system aimed to reduce time for simulating large circuit. This method offers promises to reduce cost substantially.

To accelerate circuit analysis, we used three approaches. In the first approach, we modified algorithms to adapt GPU programming strategies, which are suitable for circuit analysis and remove dependency of data. In the second

Electronic supplementary material The online version of this article (doi:https://doi.org/10.1007/s42452-020-2667-6) contains supplementary material, which is available to authorized users.

Shital V. Jagtap, svjagtap@gmail.com; Y. S. Rao, ysrao@spit.ac.in | ${ }^{1}$ Ramrao Adik Institute of Technology (R.A.I.T.), Navi, Mumbai, India. ${ }^{2}$ Sardar Patel Institute of Technology (S.P.I.T.), Andheri, Mumbai, India. 
approach, we redesigned mathematical functions used in the circuit processing. For example, we redesigned LU decomposition algorithm to execute in parallel. Many simple mathematical functions such as arithmetic operations, logical operations, finding maximum, and others are executed in parallel, with the condition that it is executable on large dataset. In the third approach, we used machine learning. We classified circuit analysis functions based on uniformity in components and methods. Clusters are formed based on suitability for parallel processing.

Figure 1 shows categories of operations for the entire flow system. All operations are executed serially except compute intensive. First block in Fig. 1 indicates input files and libraries used by the system. The netlist file, which is the input for this system, contains circuit description. As per NGSPICE simulator flow, some operations such as netlist parsing and output display have not been updated.

Second block indicates parsing of netlist to understand and store relationship between circuit components and its parameters. Inbuilt application and mathematical libraries parse netlist input file. They find connection, input, and output flow of electronic components and store the data in the structured instances. Circuit variables are created with default or derived values.

Third block indicates operations and GPU strategies used for parallel execution. Clustered heavy calculations and dataset are added in kernel and GPU memory, respectively. Optimization is applied on kernel to utilize GPU processors effectively. Reduction, avoiding warp/ thread divergence, if-else in-lining, and so on are some of the strategies to optimize GPU kernel. Once data are copied in GPU memory, processors read data in parallel. For operational analysis, data can be fetched frequently as per properties of different components but for transient analysis, data can be fetched less frequently, as the NR
(Newton-Raphson) iterations are repeated on data either available in memory or generated in previous iterations.

Currently GPU contains many general computing methods besides having graphics libraries. Advanced GPU is empowered with on-chip memory and thousands of processing cores. For example, NVIDIA Tesla V100 graphics card contains more than 5000 cores, with $32 \mathrm{~GB}$ configurations. Intel Core i7 is empowered with 8 cores that ensures 354 gigaflops [2] performance at the maximum, while Tesla V100 gives 31 teraflops [3] of performance, way ahead of Intel processor. Hence, we can say thatone single Tesla GPU offers the performance of 100 CPUs. This makes it possible for client computers to perform as a server. However, the issue with GPU cores is that it has SIMD (Single Instruction Multiple Data) property. Due to SIMD nature of GPU, sequential code developed for CPU should not be executed on GPU as it is, but optimized for GPU execution. GPU processors operate with and without synchronization with CPU. Specific to NVIDIA GPU, CUDA (Compute Unified Device Architecture) kernels are formed that specify number of threads as blocks and grids to utilize processors available in GPU.

Instead of using graphics card for playing games, there is the need of exploring computational power of GPU for research and applications for general purposes. Currently available sequential simulator algorithms are not capable for large circuit analysis. Fast circuit analysis can directly motivate researchers to design new useful electronic equipment. With this proposed technique, it can accelerate simulation performance significantly.

This paper covers machine learning-based GPU-driven circuit analysis. Section 1 includes the details of the topic. Section 2 addresses previous approaches to compare with our proposed system. Section 3 explores code design techniques for efficient SIMD GPU programming. Section 4

Fig. 1 System execution flow

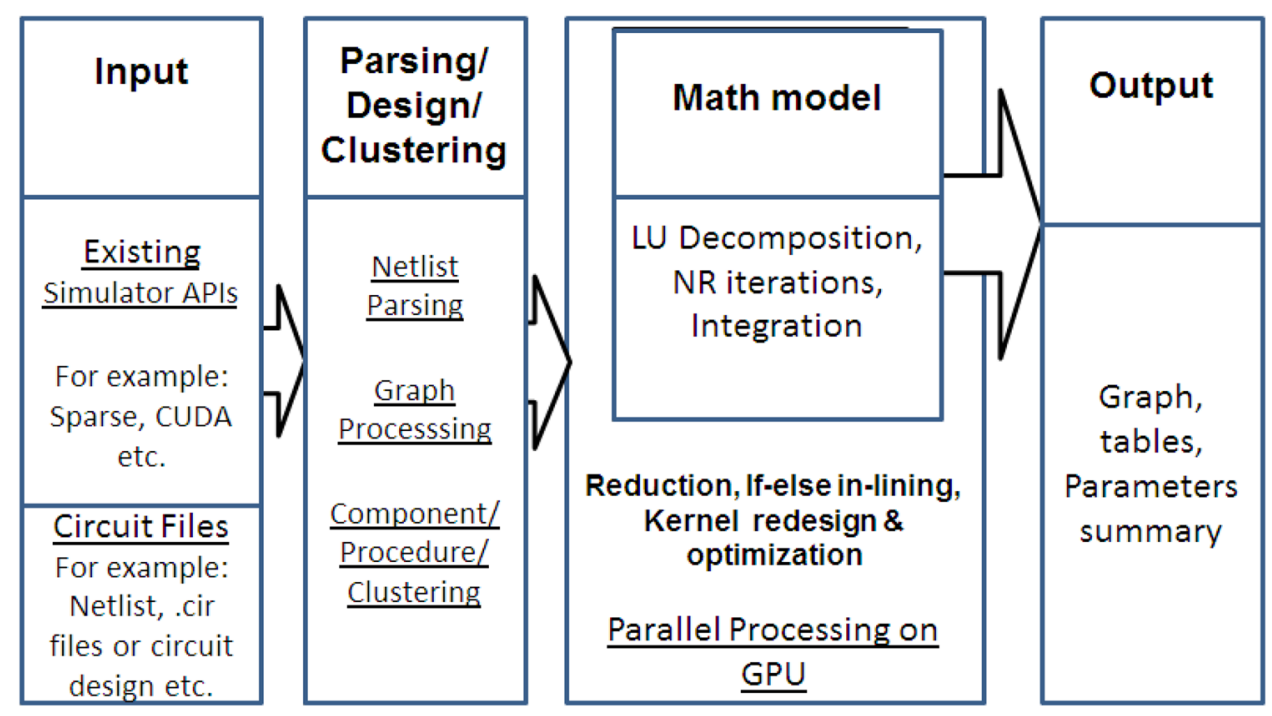

SN Applied Sciences 
gives the details of parallel version of sparse matrix solver. Section 5 explains machine learning approach for circuit analysis. Section 6 gives system performance and results. Section 7 discuses conclusion, while Sect. 8 describes future scope.

\section{Previous approaches}

Electronic Circuit Analysis (ECA) is widely used in academics and industries. Expert researchers launched some techniques such as optimizing simulator code, parallel computing using GPU, and distributed processors. These techniques have been summarized with features and limitations in Table 1.

In this experiment, parallel processing using GPU is handled with respect to compute intensive functions and parameter processing. Dynamism is induced with respect to circuit size and parameters. If these available approaches are used, it costs hugely. While in this proposed technique, the researchers have considered advantages of all the available technologies and proposed a new machine learning-based automated approach that uses computational power of GPU for execution.

\section{Code redesign techniques for GPU}

Generally, we write sequential codes that contain many if-else statements, looping, and conditional/unconditional jump statements. Such codes are not suitable for SIMD GPU architecture. So we have to modify the codes that are suitable for SIMD parallel architecture. Any sequential application code cannot be executed as it is on parallel device. Some common redesigned strategies have been used and some have been modified that adapt themselves to circuit analysis. Redesigned strategies are applied on cluster of components and methods to execute on parallel device. Figure 2 shows that resistor cluster instances and load method cluster instances are applied to many threads to execute in parallel.

Warp size is always 32. Number of threads is launched as per instance of data items available. Many threads are executed and data are processed simultaneously. Kernel code optimization is required in data storage/access through memory, number of resources such as thread, avoiding divergence, and so on.

Parallel reduction is applicable on operation on set of numbers in parallel $[25,26]$. Operation must follow commutative or associate property. For example, it takes $\mathrm{N}>1$ values and returns a single value like summation of set of numbers. Generally, in these operations data dependency is less, so it can be executed in parallel. Figure 3a shows example for finding maximum from set of eight numbers. Three steps are sufficient to get result. It means time complexity is just $\mathrm{O}(\log \mathrm{n})$. Figure $3 \mathrm{~b}$ shows how elements are accessed for comparison and where to store result. In the first step, N/2 threads compare $\mathrm{N}$ elements. Every thread performs comparison between two elements resulting into maximum value. In the second step, N/4 threads and subsequent number of threads decrease to half in next iteration [27].

Kernel is the function that contains lines of code and is executable on GPU. Hence, it should be selected carefully. Lines of instructions are applicable on few data items or it may need different parameters. If numbers of lines are more, it can be called big kernel, else it is considered a smaller one. Kernel should be big enough, so that its data are available in the memory. At the same time, kernel should be small enough, so that kernel execution time is sufficiently more than the time required for data transfer through and from memory. If-else statement contains two different code blocks depending on condition. It causes thread divergence within warp. It is time consuming even if synchronization is provided. So we can use if-else inlining concept. Consider the following example given in Sample 1 to illustrate if-else in-lining concept.

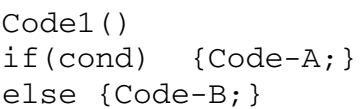

is converted to

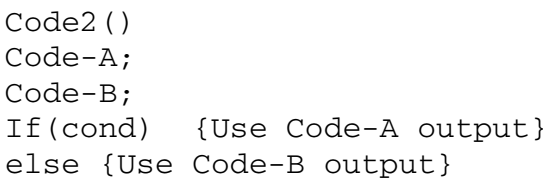

Sample 1: if-else in-lining example

In if-else in-lining, we get acceleration that is approximately equal to the execution time for smaller lines of code (from Code-A or Code-B) minus the time required to execute condition in if-statement.

- If numbers of Code-A and Code-B lines vary by hundreds of lines, then only the use of if-else in-lining is significant (in micro-seconds, for our example circuit).

- If numbers of lines are less than that, then we can use normal if-else statement.

If the threads from same warp follow different paths, then all the threads execute themselves serially reducing the performance drastically. So warp divergence in CUDA kernels should always be avoided for performance. Sample 2 has been used as an illustrative example for warp/ 
Table 1 Previous approaches

\begin{tabular}{|c|c|}
\hline Technique [citation] & Features and limitations \\
\hline $\begin{array}{l}\text { GPU with previously available technique to accelerate large circuit } \\
\text { simulation on GPU [4] }\end{array}$ & $\begin{array}{l}\text { Transistor model acceleration using if-else in-lining coalesced } \\
\text { memory access and optimum kernel size strategy } \\
\text { As it was specific to transistor model only, several researchers } \\
\text { extended this idea to various circuit acceleration proposals }\end{array}$ \\
\hline $\begin{array}{l}\text { Event-driven and gate-structure partitioning-based circuit accelera- } \\
\text { tion }[5,6]\end{array}$ & $\begin{array}{l}\text { Considers those gates that receive event trigger } \\
\text { Whole circuit is not processed. Saves execution time } \\
\text { Partitioned circuit processing in parallel is possible } \\
\text { Design level partitioning needs extra effort and memory }\end{array}$ \\
\hline $\begin{array}{l}\text { Blocking LU decomposition using FPGA [7], pivoting operations on } \\
\text { FPGA [8], scalable block LU decomposition technique [9] }\end{array}$ & $\begin{array}{l}\text { LU factorization is performed using FPGA algorithm on large sparse } \\
\text { matrices } \\
\text { Pivoting reduction is applied using FPGA } \\
\text { Compared to FPGA system, GPU technique is adaptable and cost } \\
\text { effective } \\
\text { Approach is challenging to embed in some simulators }\end{array}$ \\
\hline Fast memory level for LU decomposition on GPU [10] & $\begin{array}{l}\text { GPU memory levels are utilized efficiently to manage memory access } \\
\text { of GPU to accelerate LU decomposition [10] } \\
\text { This technique can be extended to combine many others to acceler- } \\
\text { ate speed }\end{array}$ \\
\hline Solvers like NICSLU, PARDISO $[11,12]$ & $\begin{array}{l}\text { NICSLU uses column-level dependency graph to propose the task [7] } \\
\text { Dependency is taken from the symbolic structure of the matrix factors } \\
\text { Elimination tree represents dependency among other factors } \\
\text { Techniques such as numerical update, pruning, and pivoting are used } \\
\text { for matrix stability } \\
\text { These steps can consume more time in matrix operations }\end{array}$ \\
\hline Pivoting reduction for LU sparse solver [12-15] & $\begin{array}{l}\text { Modified version of traditional solvers can be executed in parallel } \\
\text { In large circuit, matrix column dependency causes slower simulation }\end{array}$ \\
\hline KLU data structures and algorithm [16] & $\begin{array}{l}\text { Gives stability in matrix processing } \\
\text { For small circuit simulation, it proves to be slow but has been adopted } \\
\text { by many simulators such as NGSPICE }\end{array}$ \\
\hline Runge-Kutta integrators using GPU [17] & $\begin{array}{l}\text { Parallel integration execution } \\
\text { Can be extensively used in simulator application }\end{array}$ \\
\hline Distributed approach to solve sparse matrices [18] & $\begin{array}{l}\text { GPU cluster execution is possible } \\
\text { Takes extra time to allocate resources among systems and is costly as } \\
\text { compared to GPU-based system }\end{array}$ \\
\hline Various algorithms of LU decomposition on GPU [19] & $\begin{array}{l}\text { Effective strategies to implement LU technique on GPU } \\
\text { Left looking algorithm has been proved best for parallel implementa- } \\
\text { tion on GPU }\end{array}$ \\
\hline GPU-based LU decomposition algorithm for small matrices [20] & Suitable for small matrices and has to partition large matrices \\
\hline LU decomposition algorithm given for dense matrices [21] & $\begin{array}{l}\text { Is appropriate for dense matrices } \\
\text { Circuit matrices are almost sparse, although we can even use them }\end{array}$ \\
\hline GLU library [22] & $\begin{array}{l}\text { Dynamically assigns GPU (blocks and wraps) based on number of } \\
\text { columns in matrices. It properly balances the computing resources } \\
\text { This feature can be extended to other solvers on GPU }\end{array}$ \\
\hline Circuit partitioning [23] & $\begin{array}{l}\text { Circuit is partitioned into sub-circuits and mapped to GPU } \\
\text { It helps in using minimal memory requirements } \\
\text { Partitioning strategy needs to be decided to satisfy all circuit con- } \\
\text { straints }\end{array}$ \\
\hline GPU-managed kernel launch technique [24] & $\begin{array}{l}\text { To save time for launching and copying, batch and pipelined right } \\
\text { looking algorithms have been proposed } \\
\text { To group all the operations with respect to data available in GPU } \\
\text { memory }\end{array}$ \\
\hline
\end{tabular}

thread divergence. But in actual simulator coding, these issues are handled properly. Sample 2 gives the example for code modification to avoid thread and warp divergence. In the following code, it shows that alternate threads are using different paths. From the same warp neighbouring threads, performance is not same and causes thread or warp divergence.

\section{SN Applied Sciences}




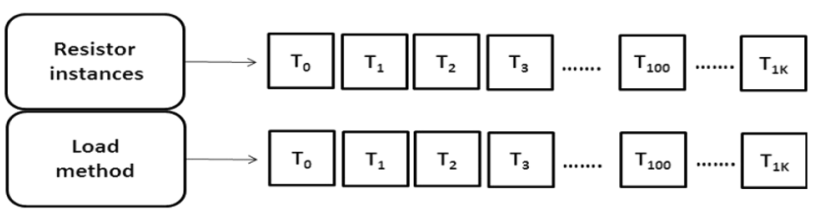

Fig. 2 Code structure for execution

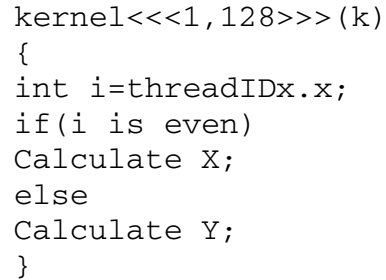

is converted to

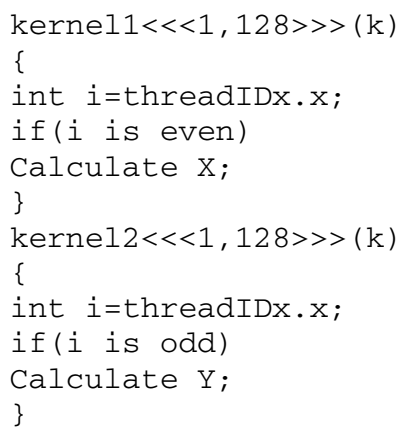

Sample 2: warp/thread divergence example

We created two different CUDA kernels for even and odd calculations each, as kernel launch overhead is very small, just of few nanoseconds.

Specific to circuit analysis, one major change has been implemented. Instead of copying data one after the other, structure array is used for component parameters. As different memory levels are available in GPU with different speed and size, data needed more frequently should be copied in shared memory.

Page-locked host memory for data transfers can also be used. GPU access data from pinned memory mean GPU memory. CPU malloc() function is used to host data, as it is in CPU pageable non-pinned memory. When cudaMemcpy() is called, the CUDA driver has to first copy the data (memcpy) from non-pinned memory pointer to the internal pinned memory pointer, and then the host GPU memory access can be invoked. If host memory is allocated with cudaMallocHost and the data are initialized directly, the driver does not have to copy the data (memcpy) from pageable into pinned memory before memory access. It accesses directly, making it faster as it reduces copying time.

Coalesced memory access optimizes time to read and write data. Fast shared memory, constant data memory, and $1 \mathrm{D} / 2 \mathrm{D}$ texture memory can be used according to the need. Page-locked host memory is also useful. cudaMalloc() and cudaFree() are costly operations, so we minimized by reusing the allocations. Structure arrays are reorganized to occupy minimum memory. We used asynchronous data copy, wherever possible.

In this GPU-based system, data are copied in structure instance instead of KLU structures. As component count increases, copy time also increases. Copy time is directly proportional to the number of components. For example, if 10 components are processed, 10 units of time is required in parallel.
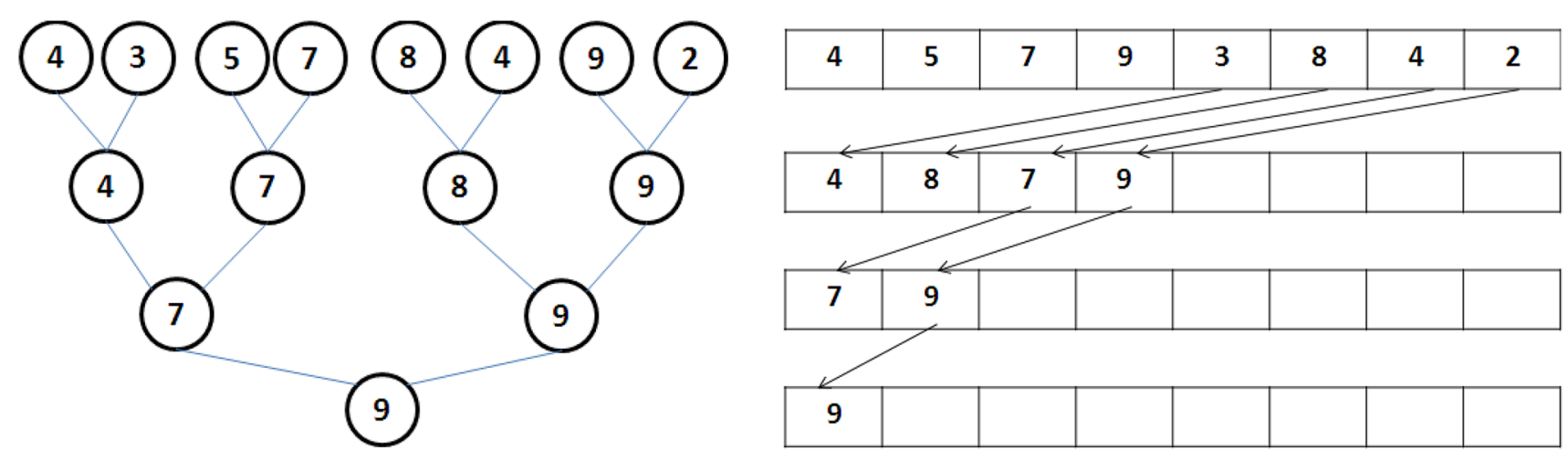

(a) Numbers to compare

(b) Memory map of numbers

Fig. 3 GPU thread and memory access 


\section{Parallel sparse matrix solver}

To accelerate speed of circuit simulation, first it is important to analyse time required for mathematical functions. Also these functions should be compute intensive to convert it into SIMD type of kernels. Here we find that out of total circuit analysis time, approximately $36 \%$ of time is used in solving sparse matrices generated for nodal analysis of a circuit.

Default dense matrix library of GPU is not convenient to solve nodal matrix as it is sparse. In the factorization, step diagonal elements are used in denominator of division operation. So diagonal element must be non-zero. To get stable result, we need some pre-processing. Pre-processing operations used are like symbolic analysis to find non-zero pattern in matrix elements. Symmetric pruning is required to find structural symmetry in the matrices. Zero element should be replaced with non-zero element. Depth-first search (DFS) is useful for topological ordering of elements. Ordering also helps in finding permutation matrices. Permutation of rows or columns or both are used to avoid zero in diagonal of matrices.

Pre-processed circuit matrix is then factorized using Gaussian elimination, i.e. factorization. Left looking LU factorization is more appropriate for parallel conversion as the columns of lower and upper matrix calculation are more independent elements. Therefore, it has been used selectively for parallel execution. Both upper and lower triangular matrices are processed column by column.

Equations (1) and (2) show operations involved and are executed in several iterations. Here ' $a$ ' is the matrix element, whereas ' $m$ ' is the multiplication factor generated from different column elements, ' $j$ ' is the column index, and ' $i$ ' is the row index.

$m_{i, k}=\frac{a_{i, k}}{a_{k, k}}$

$a_{i, j}=a_{i, j}-m_{i, k} a_{k, j}$

Here variable index range is like $k=1$ to $n$ and $j=k+1$ to $n$.

Fig. 4 LU Decomposition

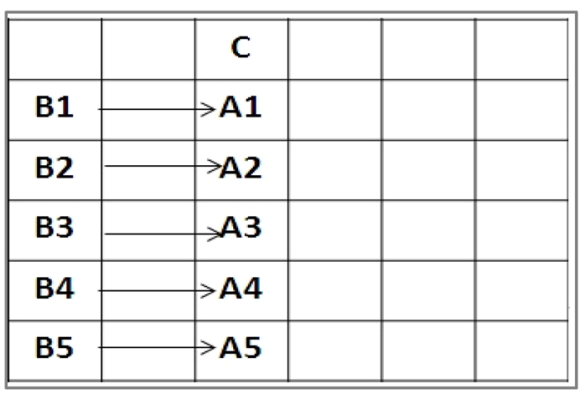

(a) Matrix column dependency
In algorithmic form, sequential LU decomposition algorithm is shown in Algorithm 1 and its worst-case execution time complexity is $\mathrm{O}\left(\mathrm{N}^{3}\right)$. Here ' $\mathrm{N}$ ' indicates approximate component count (or sometimes nodes).

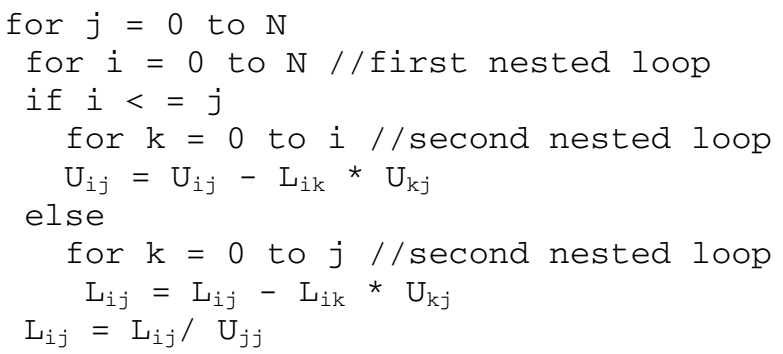

Algo 1. LU decomposition algorithm (Sequential)

It updates matrix data column by column to generate lower and upper triangular matrices. Here each succeeding column is dependent on previous columns. For small circuit, matrix size is small, but for large circuit ' $\mathrm{N}$ ' can exceed 1000. To process big matrices, GPU-based matrix operations are found to be very effective. Simplified operation for LU decomposition is shown in Fig. 4 and Eq. (3). In LU decomposition, column element calculations are independent of each other. Each succeeding column is dependent on data available in previous columns. Figure $4 a$ shows that to do the calculation for column ' $A$ ', we need values from column ' $B$ '. Column ' $B$ ' elements are the inputs to many cores of GPU to calculate elements of column ' $A$ ' as shown in Fig. $4 b$.

$A_{i}=A_{i}-C * B_{i}$.

$\mathrm{P} 1$ processor reads data $\mathrm{B} 1$, performs calculation, and the result is stored at A1. Similarly, other processors work on data available at consecutive locations. Modified LU algorithm is shown in Algorithm 2. In this algorithm, ' $j$ ' variable indicates thread number that points to the column of matrices.

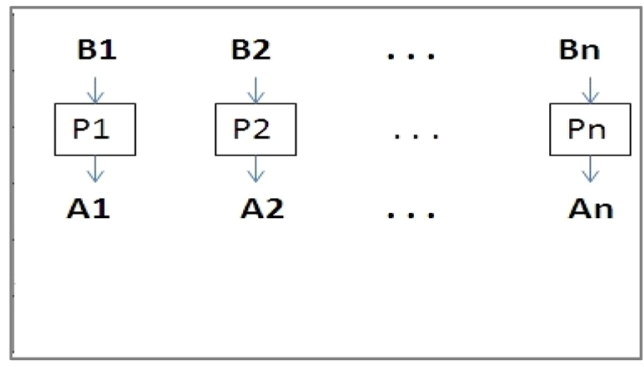

(b) Column access in parallel 


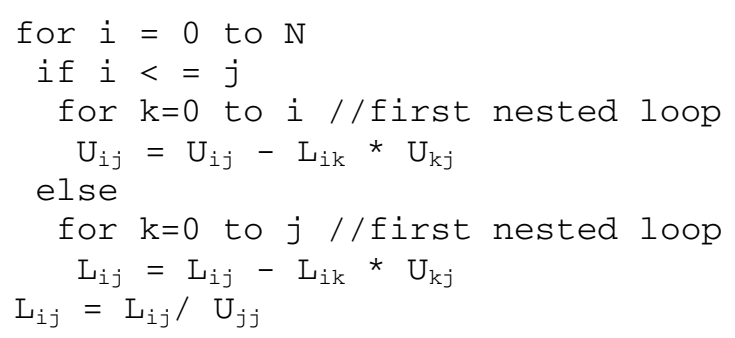

Algo 2. LU decomposition algorithm (Parallel)

For parallel operation through GPU, data are required to process and launch ' $\mathrm{N}$ ' threads that perform operations in parallel. As the succeeding column is dependent on previous column, execution of two or more columns in parallel is not possible. One column of matrices is assigned in the kernel and executed at a time on parallel cores. One thread calculates one element of column. Proper synchronization is required to avoid any data-dependent operation. Worst-case complexity of parallel LU algorithm is $\mathrm{O}\left(\mathrm{N}^{2}\right)$. Constants or fixed data such as diagonal of lower triangular matrices can be initialized prior to the operation to save execution cycles. The 'if' statement has been tried to minimize as far as possible as it may cause warp or thread divergence. Proper initialization of $L$ and $U$ matrices can reduce some of the 'if' statements. GPU contains on-chip levels of memory with different access speed. Shared memory accelerates the speed as compared to global memory access in GPU.

Finally, using backward and forward substitutions, it generates parameter values.

\section{Machine learning approach}

Machine learning technique is useful in training the model. In circuit simulation, circuit may have many different components to process. Hence, it is not the solution to develop different strategies for different circuits. We have developed machine learning strategy to train simulator to select functions that can execute in parallel. It gives us automated model for parallel kernel execution. It also helps us in inducing dynamism to select component runtime for parallel execution.

As different circuit components have different parameters such as resistance, capacitance, and static current, we need a technique to wrap all components having same set of parameters in one group. Classification and clustering [27] are helpful to classify the features by identifying variance and group them using clustering. Millions of parameters are classified in large circuit and grouped in cluster. Depth-first search (DFS) algorithm is helpful here to identify components, their connection and parameters. $\mathrm{K}$-means clustering is also helpful to decide cluster centroid with respect to the most frequent components of circuit.

After netlist parses components, its execution methods are clustered and then compute intensive methods are executed through GPU. This process is shown in Fig. 5 .

\subsection{Component clustering}

Similar components are clustered together. Advantage of component clustering is that the same type of processing is applicable to all the instances of clusters. Processing includes parameter initialization, setup, loading, and so on. Also, procedure to calculate conductance is the same. If components are same, sub-operations are exactly the same; hence cluster operations are exactly the same. It helps in accelerating speed as compared to processing with different components in parallel.

Property structure, component properties count, and data type are the parameters used to perform classification and clustering. Variance is calculated using Eq. (4). Appropriate weights are assigned to each feature vector or property.

Variance $=\sum \frac{\left(x-x^{\prime}\right)^{2}}{n}$.

Variance of all similar components is almost zero. For dissimilar components, variance is non-zero large value. For example, resistance and capacitance have different weights and different mathematical formulae for calculating conductance. Hence, as per variance formula, it can generate non-zero value. If this non-zero difference value is greater than 100, we can add resistor and capacitor in different clusters. We can set desired threshold to accept it in clusters or not.

Let $C$ be the component, $M$ the method, $X$ the parameter with data type $D$, then

$C \in\{$ Resistor, capacitor, transistor, Op-amp, diode, FET, BJet, ........\}

$M \in$ \{setup, load, LUfactor, Nliter, spSolve, spfactor, parse, ........

$X \in\{$ Resistance, impedance, capacitance, current, drain, ......... $\}$

$D \in\{$ double, float, int, double-array, structure, structurearray, pointer, ...... $\}$.

It forms the component clusters. It means all the resistors are wrapped in one cluster or all capacitors are wrapped in one cluster. In component clustering, intracluster similarity is very high and inter-cluster similarity is very low. 
Fig. 5 Clustering and mapping to GPU

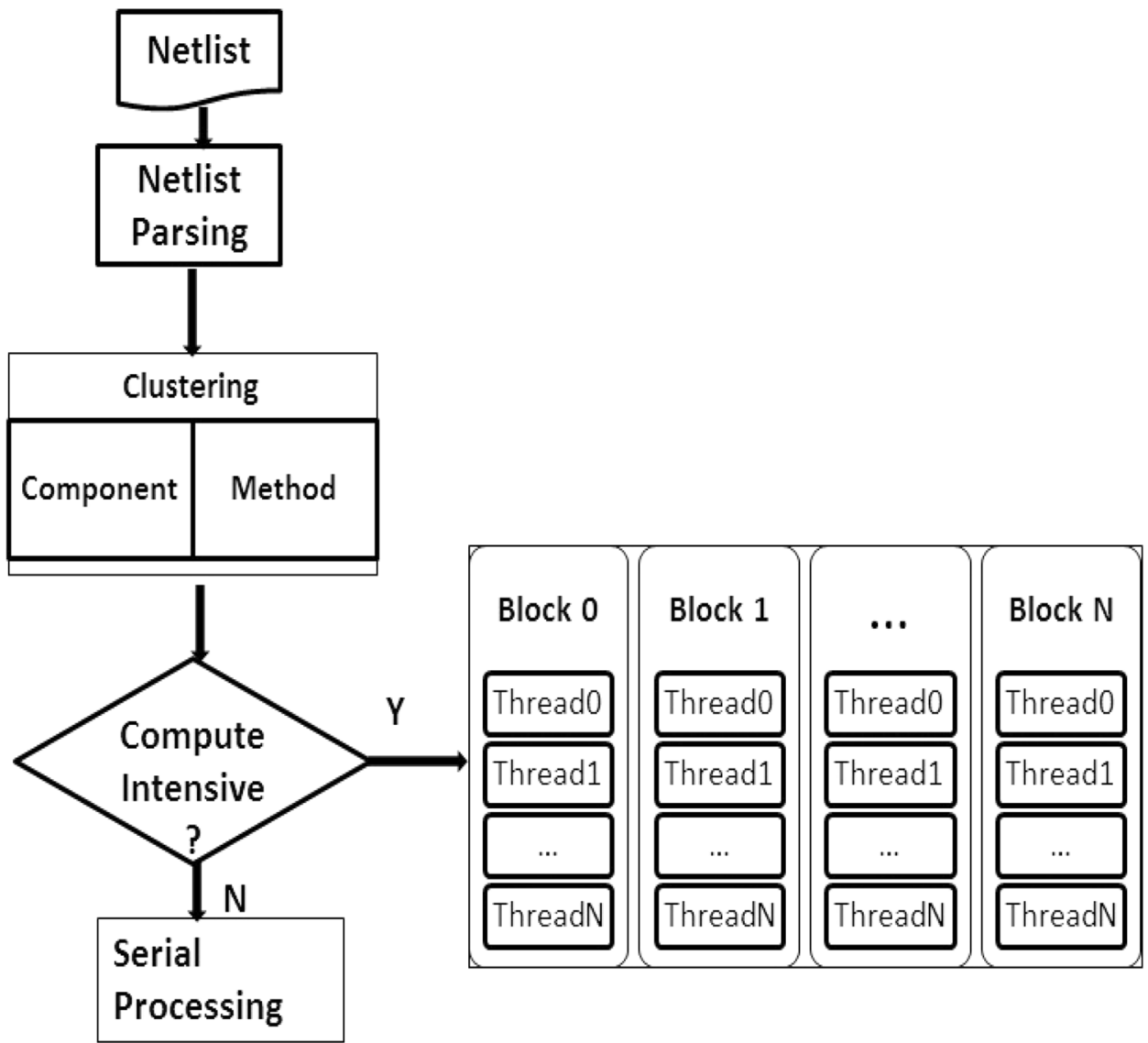

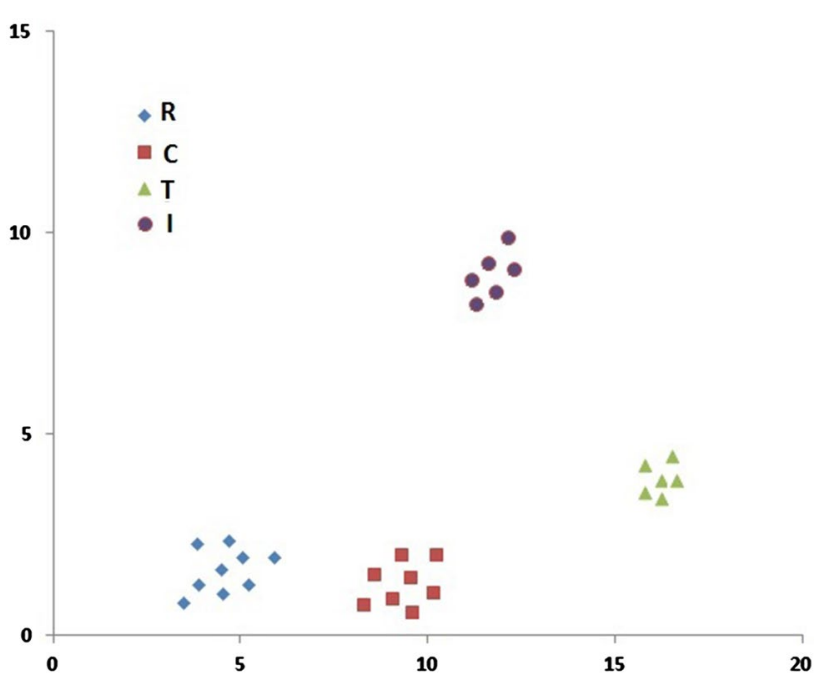

Fig. 6 Component clusters

$R \in\left\{R_{1}, R_{2}, R_{3} \ldots R_{n}\right\}$.

$T \in\left\{T_{1}, T_{2}, T_{3} \ldots T_{n}\right\}$.

Figure 6 shows clusters of resistor $(R)$, capacitor $(C)$, inductor $(l)$, and transistor $(\mathrm{T})$.

If the parameters processing for different components are same, they are also grouped in one cluster. For example, many parameters and formulae of different voltage sources are same, so small variance properties can be grouped together.

V $\{$ VCC, VPP, VSRC $\}$.

Circuit raw data contain millions of parameters of all components that vary periodically. There are various types of parameters such as some are fixed, some are independent, and some are derived. Data items are divided into different sets such as component features and method features. Table 2 shows component feature vectors.

\subsection{Procedure clustering}

Procedure clustering is more complex and time consuming than the component clustering. However, procedure clustering is more suitable for GPU computing, as many thread instance formations are possible due to SIMD nature of GPU. Component analysis involves many processing methods such as initialization, load, mathematical operations, and so on. Also, many components have common and simple processing methods such as initializing parameters with value ' 0 ' or some temperature-based calculation. Such common methods can be grouped together 
Table 2 Feature vectors for components

\begin{tabular}{|c|c|c|c|c|c|c|c|c|}
\hline Component & $\mathrm{F} 1$ & $\mathrm{~F} 2$ & F3 & $\mathrm{F} 4$ & F5 & F6 & F7 & F8 \\
\hline Resistor & Resistance & & Current & Temperature & Thermal\noise & & & \\
\hline Capacitor & & Capacitance & Current & Temperature & Thermal\noise & Charge & & \\
\hline Transistor & & & & Temperature & Thermal \noise & & $\begin{array}{l}\text { Drain } \\
\text { node }\end{array}$ & Drain current \\
\hline
\end{tabular}

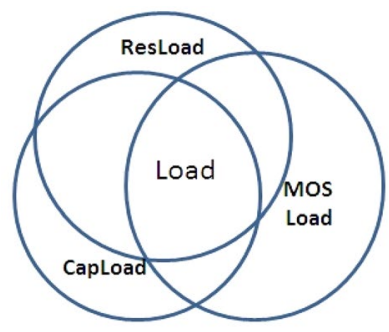

(a) Load method cluster

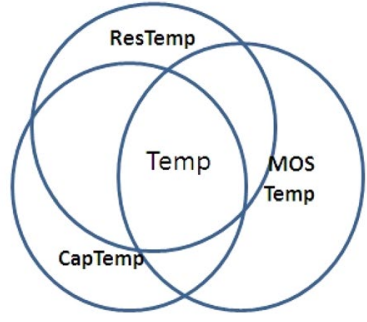

(b) Temperature method cluster
Fig. 7 Method clusters

in one procedure cluster. Initialization, load, setup, and the methods that do not have any data dependency can be grouped together. We try to increase intra-cluster similarity compared to inter-cluster similarity. Figure 7 shows the clusters of commonly uniform executable methods. Table 3 shows procedure feature vectors that are required for procedure clustering.

There are limitations on GPU memory size and extra time is needed in loading and unloading all the parameters. Hence, if serial time is much more as compared to parallel cluster execution time plus loading time, it is suitable to make it parallel. Once clusters are formed, these are executed on GPU. GPU programming optimization strategies are applicable to modify and redesign the code.

\section{Results}

Parallel version with KLU is compatible with NGSPICEan open source simulator. However, in this experiment, sequential code has been used to embed proposed changes. NVIDIA GeForce $840 \mathrm{M}$ graphics card is used that has 384 cores and its compute capability is 5.0 with $2 \mathrm{~GB}$ graphics card memory. One high-end GPU launched recently like Tesla V100 has also been used for checking performance. Tesla V100 is available with 32 GB memory. Ubuntu 14.04 version was used for NGSPICE coding. We got acceleration in two types of analysis: first is operational and the other is transient analysis. Netlist parsing time is constant, so it has not been included in time measured. Clustering plus actual execution was considered. Time taken in copying data was also considered. In transient analysis, more than thousand iterations were considered to get all possible time instances. Percentage speed gain is calculated using Eq. (5).

Percentage speed gain $=100 * \frac{(\text { serial }- \text { parallel time })}{\text { parallel time }}$.

Execution time varies in every execution cycle, and hence least execution time was considered for this experiment.

In operational analysis, circuit with at least hundreds of components was considered. For transient analysis, two types of analysis have been tested: (1) operational analysis and (2) transient analysis. Large and small circuits were considered for operational and transient analyses, respectively.

Table 4 shows serial and parallel execution time for operational analysis of some of the netlist having $100+$ components or sub-circuits if executed with Geforce GTX M840. It also shows parallel execution time of operational analysis of same sub-circuits if executed with Tesla V100. In both the scenarios, it shows that serial execution time is more than parallel execution time.

Table 3 Feature vectors for methods

\begin{tabular}{|c|c|c|c|c|c|c|c|}
\hline Methods & $\mathrm{F} 1$ & $\mathrm{~F} 2$ & F3 & F4 & F5 & F6 & F7 \\
\hline Load & Assignment to 0 & Add/sub & Mult/div & $\log / \operatorname{Exp}$ & Integration & Complex & \\
\hline Setup & Assignment to 0 & Add/sub & Mult/div & $\log / \operatorname{Exp}$ & Integration & Complex & \\
\hline Temperature & Assignment to 0 or default & Add/sub & Mult/div & $\log / \operatorname{Exp}$ & Integration & Complex & $\begin{array}{l}\text { Thermal } \\
\text { noise cal- } \\
\text { culation }\end{array}$ \\
\hline
\end{tabular}


Table 4 Serial and parallel execution time of operational analysis on M840 and Tesla V100

\begin{tabular}{|c|c|c|c|c|c|c|}
\hline \multirow[t]{2}{*}{ Netlist } & \multirow[t]{2}{*}{ Circuit } & \multirow[t]{2}{*}{$\begin{array}{l}\text { Serial execution } \\
\text { (micro-sec) }\end{array}$} & \multicolumn{2}{|c|}{$\begin{array}{l}\text { Parallel execution on GTX } \\
\text { M840 }\end{array}$} & \multicolumn{2}{|c|}{$\begin{array}{l}\text { Parallel execution on } \\
\text { Tesla V100 }\end{array}$} \\
\hline & & & $\begin{array}{l}\text { Time in } \\
\text { micro-sec }\end{array}$ & $\begin{array}{l}\text { Speed gain } \\
(\%)\end{array}$ & $\begin{array}{l}\text { Time in } \\
\text { micro-sec }\end{array}$ & $\begin{array}{l}\text { Speed } \\
\text { gain } \\
(\%)\end{array}$ \\
\hline 1 & AD629B & 227 & 195 & 16 & 183 & 24 \\
\hline 2 & AD1580 & 285 & 235 & 21 & 224 & 27 \\
\hline 3 & AD584 & 337 & 287 & 17 & 269 & 25 \\
\hline 4 & SSM2212 & 361 & 310 & 16 & 284 & 27 \\
\hline 5 & AD587 & 487 & 396 & 23 & 375 & 29 \\
\hline 6 & PM1012 & 1112 & 901 & 23 & 846 & 31 \\
\hline 7 & AD5144 & 2680 & 2197 & 22 & 2021 & 32 \\
\hline 8 & AD588 & 7353 & 6157 & 19 & 5865 & 25 \\
\hline
\end{tabular}

*Circuit netlist files reference: https://www.analog.com/en/design-center/simulation-models/spice -models.html
As number of components in the circuit increases, parameter copying time from CPU to GPU memory also increases. However, average speed gain is more than $16 \%$ using cost effective GPU. Figure 8 shows a comparison of serial and parallel executions.

High-end GPU gives better performance as number of processing cores and available GPU memory is more. As more memory is available on chip, data swapping reduction at frequent pace is possible. Also, it can store big lookup tables. Many data items are processed in parallel by utilizing as many processors as available. Therefore, average speed gain is more as compared to low-end GPU. For small circuit, low-end GPU is sufficient to process circuit parameters but for large circuit, high-end GPU can accelerate speed.

Table 5 shows execution time of transient analysis on M840 and Tesla V100, respectively. All the fundamental circuit netlist such as JFET amplifier and Bridge rectifier having fewer components have been considered. Here, execution time is also measured in NGSPICE version having KLU data structure [28]. Circuit is tested for thousands of transient iterations. It shows that basic
Fig. 8 Time taken in serial vs parallel operational analysis

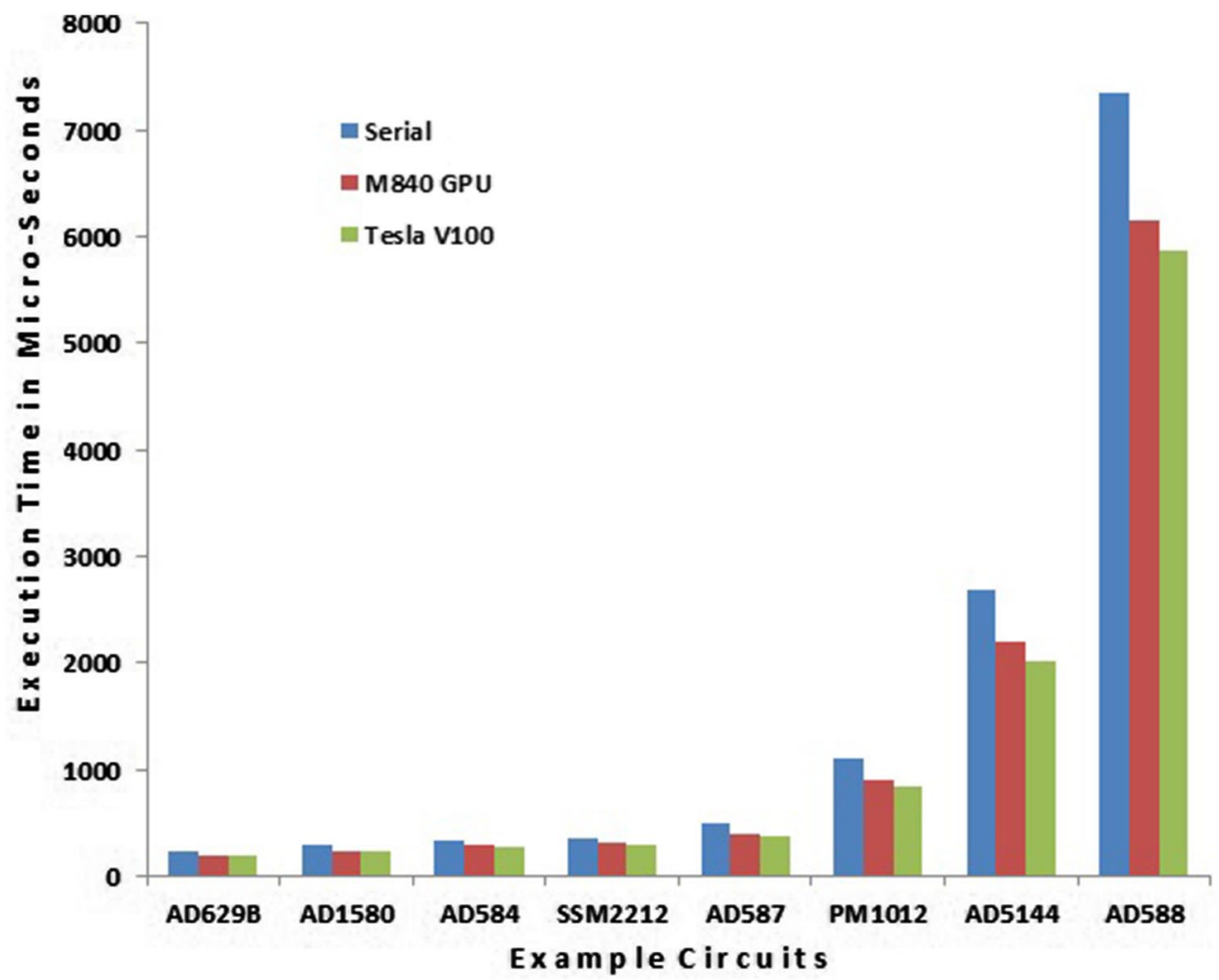


GPU strategies and clustering approach accelerate circuit processing at least by $49 \%$ and increases subsequently for more iterations. Figure 9 shows comparison between transient execution time of NGSPICE with KLU, GTX M840 and Tesla V100.

\section{Discussion and conclusions}

In sequential circuit analysis, analysis time increases exponentially if we embed more components. But in parallel processing, average analysis time increases linearly as we add more components in the circuit. Time taken for data transfer from CPU to GPU and GPU to CPU memory, clustering, weight assignment, and so on increases with increase in number of components affecting speed.

Table 5 Transient analysis time comparison

\begin{tabular}{|c|c|c|c|c|c|c|c|}
\hline \multirow[t]{2}{*}{ Netlist } & \multirow[t]{2}{*}{ Circuit } & \multirow[t]{2}{*}{ No. of iterations } & \multirow[t]{2}{*}{$\begin{array}{l}\text { With KLU parallel } \\
\text { execution ( in sec) }\end{array}$} & \multicolumn{2}{|c|}{$\begin{array}{l}\text { Without KLU Parallel execu- } \\
\text { tion on GTX M840 }\end{array}$} & \multicolumn{2}{|c|}{$\begin{array}{l}\text { Without KLU Parallel execu- } \\
\text { tion on Tesla V100 }\end{array}$} \\
\hline & & & & Time in sec & Speed gain (\%) & Time in sec & Speed gain (\%) \\
\hline 1 & AC sine wave voltage & 10,000 & 6.38 & 3.75 & 70 & 3.31 & 92 \\
\hline 2 & RC circuit & 1008 & 0.85 & 0.58 & 47 & 0.57 & 49 \\
\hline 3 & Full wave bridge rectifier & 10,000 & 12.54 & 8.23 & 52 & 7.60 & 65 \\
\hline 4 & Common source JFET amplifier & 10,000 & 8.71 & 4.26 & 104 & 3.96 & 119 \\
\hline 5 & Integrator with square wave input & 2520 & 2.115 & 1.4875 & 42 & 1.29 & 63 \\
\hline
\end{tabular}

Fig. 9 Transient analysis for serial vs parallel executions time

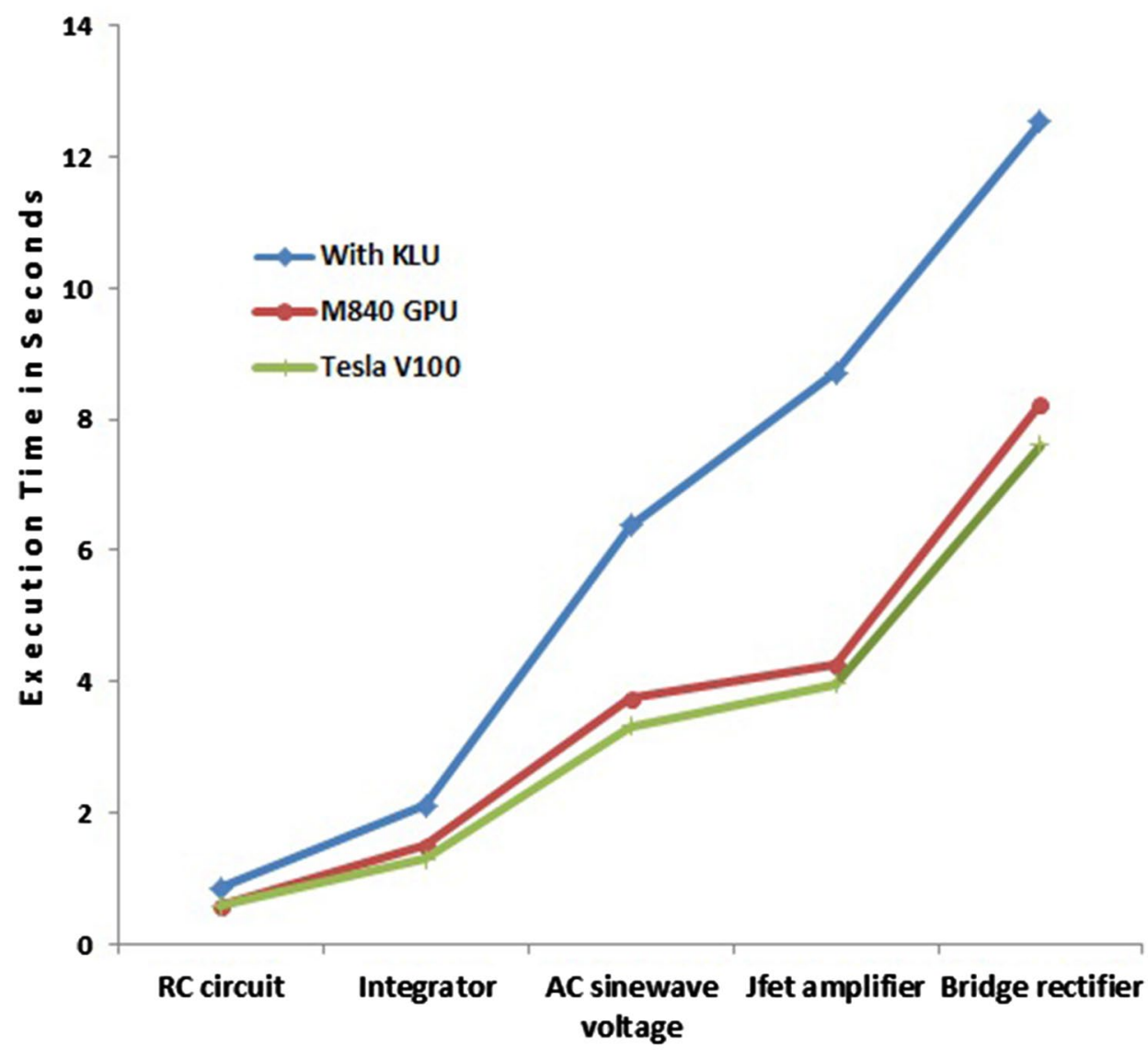

Example Circuits 
When we considered different circuits for analysis, we found that as number of components and type of component vary, processing also varies. Similarly, cluster size and cluster formation time also vary. However, we still found that operational analysis has been accelerated by more than $15 \%$, while high-end GPU can accelerate it more, up to $24 \%$.

In operational analysis, only one Newton-Raphson iteration is required, so that clustering is executed at one attempt only. In transient analysis, many Newton-Raphson iterations are required but clustering procedure is not repeated. Also, some initialization operations are common and not repeated. So speed gain in transient analysis is good as compared to operational analysis. In transient analysis, speed gain (in comparison with serial analysis) increases as we increase number of iterations. Using new generation GPUs, we can speed up to $49 \%$.

\section{Future scope}

Drawback of the current system is low memory in GPU. Multi-GPU would be required in future scope to gain high memory aimed to reduce time taken for data transfer. As processor count increases, processing time spent will also decrease. In using multi-GPU, algorithm should be redesigned to utilize memory and processor available on multiple GPU. Also map-reduce technology should be adapted to divide and map data items on available cores from GPUs.

Some optimized machine-deep learning mathematical functions are available in modern tensor cores. It will surely help in minimizing clustering and classification timing. However, redesigning of those functions are required for suitability for circuit matrices. Research is also required on use of upcoming deep learning algorithm on circuit analysis automated functions.

Research is also feasible to adopt FP16 system for data storage. FP16 will minimize memory requirement reducing time to copy data. Hence, clustering operation time and training time will also reduce.

\section{Compliance with ethical standards}

Conflict of interest On behalf of all authors, the corresponding author states that there is no conflict of interest.

\section{References}

1. Jagtap SV, Rao YS (2013) Clustering and parallel processing on GPU to accelerate circuit transient analysis. International
Conference on Advanced Computing Networking and Informatics, Springer.https://doi.org/10.1007/978-981-13-2673-8

2. https://www.intel.in/

3. https://www.nvidia.com/en-us/data-center/v100

4. Gulati K, Croix JF, Khatri SP, Shastry R, Fast circuit simulation on graphics processing units, IEEE XPlore, Available:https://doi. org/10.1109/ASPDAC.2009.4796514

5. Chatterjee D, DeOrio A, Bertacco V, Event driven gate-level simulation with GP-GPUs. ACM Digital Library. Available: https ://dl.acm.org/citation.cfm?id=1630056.

6. Chatterjee D, DeOrio A, Bertacco V, Gate-level simulation with GPU computing. ACM transactions on design automation of electronic systems, ACM Digital Library. Available: https:// dl.acm.org/citation.cfm?id=1970363.

7. Wu G, Dou Y, Peterson GD (2010) Blocking LU decomposition for FPGAs. 18th IEEE Annual International Symposium on FieldProgrammable Custom Computing Machines. Available: https ://dl.acm.org/citation.cfm?id=1827863.

8. Shao Y, Jiang L, Zhao Q, Wang Y (2009) High performance and parallel model for LU decomposition on FPGAs. Fourth International Conference on Frontier of Computer Science and Technology, Shanghai, China, Available: IEEE XPlore.https://doi. org/10.1109/FCST.2009.66

9. Jaiswal MK, Chandrachoodan N (2012) FPGA-based high performance and scalable block LU decomposition architecture. IEEE transactions on computers. 61(1). Available: IEEE XPlore.https:// doi.org/10.1109/TC.2011.24

10. Cupertino LF, Singulani AP, da Silva CP, Pacheco MAC (2010) LU decomposition on GPUs: the impact of memory access. IEEE XPlore Digital Library. Available: IEEE XPlore. https://ieeexplore .ieee.org/document/5645390.

11. Chen X, Wang Y, Yang H (2013) NICSLU: an adaptive sparse matrix solver for parallel circuit simulation. IEEE transactions on computer-aided design of integrated circuits and systems, 32(2). Available: IEEE XPlore.https://doi.org/10.1109/TCAD.2012.22179 64

12. Chen $X$, Wang $Y$, Yang $H, A$ fast parallel sparse solver for SPICEbased circuit simulators. CiteSeer ${ }^{X}$. Available: https://citeseerX. ist.psu.edu/viewdoc/summary?doi=10.1.1.724.4153.

13. Ren L, Chen X, Wang Y, Zhang X, Yang H. Sparse LU factorization for parallel circuit simulation on GPU. IEEE XPlore Digital Library. Available: https://ieeexplore.ieee.org/document/6241646.

14. Chen $X$, Wang $Y$, Yang $H$, An adaptive $L U$ factorization algorithm for parallel circuit simulation. IEEE XPlore Digital Library. Available: https://ieeexplore.ieee.org/document/6164974.

15. Chen X, Su D, Wang Y, Yang H Nonzero pattern analysis and memory access optimization in GPU-based sparse LU factorization for circuit simulation. CiteSeer ${ }^{\mathrm{X}}$. Available: https://cites eerx.ist.psu.edu/viewdoc/summary?doi=10.1.1.719.1828.

16. Davis T, Natarajan EP (2010) Algorithm 907: KLU, a direct sparse solver for circuit simulation problems. ACM transactions on mathematical software, 37(3). Article 36. Available:https://doi. org/10.1145/1824801.1824814

17. Murray L (2010) GPU acceleration of Runge-Kutta integrators. IEEE transactions on parallel and distributed systems, 23(1). Available: ACM Digital Library.https://doi.org/10.1109/ TPDS.2011.61

18. Sao P, Vuduc R, Li X, A distributed CPU GPU sparse direct solver. Berkeley Lab. Available: https://crd-legacy.lbl.gov/ xiaoye/ europar14.pdf.

19. Bandara HMDM, Ranasinghe DN Effective GPU strategies for LU decomposition. IEEE International Conference on High Performance Computing, HiPC. Available: https://hipc.org/hipc2011/ studsym-papers/1569512927.pdf

20. Dong T, Haidar A, Luszczek P, Harris JA, Tomov S, Dongarra J LU factorization of small matrices: accelerating batched DGETRF on 
the GPU. ACMDL Digital Library. Available: https://dl.acm.org/ citation.cfm?id=2760899

21. Galoppo N, Govindaraju NK, Henson M, Manocha D, “LU-GPU: efficient algorithms for solving dense linear systems on graphics hardware. ResearchGate. Available: https://www.researchga te.net/publication/220782520_LU-GPU_Efficient_Algorithms _for_Solving_Dense_Linear_Systems_on_Graphics_Hardware

22. Peng S, Tan SXD (2019) GLU3.0: fast GPU-based parallel sparse LU factorization for circuit simulation, arXiv:1908.00204v2[cs. DC], 2019. https://arxiv.org/pdf/1908.00204.pdf

23. Lyras G, Rodopoulos D, Papanikolaou A, Soudris D, Hypervised transient SPICE simulations of large netlists \& workloads on multi-processor systems. IEEE, 10.7873/DATE.2013.142, https:// ieeexplore.ieee.org/document/6513588

24. Lee W-K, Acharand R. Nakhla MS, Dynamic GPU parallel sparse LU factorization for fast circuit simulation. IEEE Transactions on Very Large Scale Integration (VLSI) Systems. https://ieeexplore .ieee.org/document/8430608

25. Jradi WAR, Nascimento H, Martins WS, Fast A (2018) Generic GPU-based parallel reduction implementation, IEEE Xplorer,
Symposium on High Performance Computing Systems (WSCAD), Sao Paulo. Brazil 2019:16-22. https://doi.org/10.1109/ WSCAD.2018.00013

26. Reduction reference https://developer.download.nvidia.com/ assets/cuda/files/reduction.pdf

27. Jagtap SV, Rao YS (2019) Cluster-based and GPU-driven parallel computing model to accelerate circuit simulation. Int J Innov Technol Explor Eng

28. Lannnutti F, Nanzi P, Olivieri M (2012) KLU sparse direct linear solver implementation into NGSPICE. 19th International Conference on Mixed Design of Integrated Circuits and Systems, 24-26, Poland.

Publisher's Note Springer Nature remains neutral with regard to jurisdictional claims in published maps and institutional affiliations. 